\title{
Auch im Jahr 2004 ist das Praxislabor noch wirtschaftlich
}

\author{
M. Hug, Präsident der Laborkommission des KHM
}

Korrespondenz:

Dr. med. Markus Hug Rue de la Préfecture 1 CH-2800 Delsberg
Seit dem 1. Januar 2004 gilt für das Praxislabor landesweit die Eidgenössische Analysenliste. Das heisst, dass die Betreiber von Praxislabors in den meisten Kantonen eine Reduktion der Tarife in Kauf nehmen mussten. Für viele Kantone hatte die Eidgenössische Analysenliste bereits vorher Gültigkeit. Die Adaptierung der kantonalen Tarife an die Eidgenössische Analysenliste konnte eine noch weitgehendere Tarifreduktion für das Praxislabor verhindern. Der Vorschlag des BAG verlangte zu diesem Zeitpunkt einen Taxpunktwert von 0,80. Man sollte in diesem Zusammenhang jedoch bedenken, dass die Tarife in einigen Kantonen doppelt so hoch waren wie der schweizerische Durchschnitt.

Dass das Praxislabor medizinisch und wirtschaftlich sinnvoll ist, wurde in der Studie von Prof. Felix Gutzwiller und Prof. Thomas Szucs eindrücklich dargestellt. Die Wirtschaftlichkeit für die Ärzteschaft ist eine andere Sache. Das BAG anerkennt im allgemeinen eine Rentabilität von 25 bis 30\%. Dies mag für viele Kolleginnen und Kollegen an der Grenze zur Unwirtschaftlichkeit sein, doch diese Beurteilung bleibt jedem selbst überlassen. Wir halten die jetzt gültige Abgeltung für vernünftig und meinen aber auch, dass es keinesfalls zu weiteren Tarifreduktionen kommen darf. Die heutige Regelung soll vor allem auch für Kolleginnen und Kollegen gelten, die Laboruntersuchungen im Rahmen einer sinnvollen diagnostischen Abklärung verwenden und die weniger als die in der Praxislaborstudie erhobenen durchschnittlichen 130 Analysen pro Woche durchführen.

Wir möchten daran erinnern, dass für das Praxislabor regelmässige Qualitätskontrollen obligatorisch sind. Die externen Qualitätskon- trollen ermöglichen es, gröbere Fehler in den Analysen aufzufinden. Die Einführung der internen Qualitätskontrollen, welche alle zwei Wochen vorzunehmen sind, werden zu einer deutlichen Verbesserung der Genauigkeit bei den Untersuchungen beitragen. Dieser Rhythmus scheint angesichts der Zuverlässigkeit moderner Analysen sinnvoll zu sein. In Grosslabors wird jede Methode mindestens zweimal pro Tag je im normalen und pathologischen Bereich kontrolliert, was bis zu 20\% der Analysenkosten ausmacht. Das Führen des Labortagebuchs und die Auswertung der Resultate der Qualitätskontrollen nach den Kriterien der Zusatzausbildung für das Praxislabor (Fähigkeitsausweis) ermöglichen es, zuverlässige Resultate bei seinen Analysen zu erhalten und damit einen wichtigen Beitrag zu einer qualitativ hochstehenden medizinischen Versorgung zu leisten.

Die Laborfrage wird in zahlreichen Kommissionen auf Bundesebene, deren Kompetenzbereiche sich teilweise überschneiden, diskutiert. Das letzte Wort hat jedoch das Eidgenössische Departement des Innern. Wir setzen uns beim verantwortlichen Bundesamt (BAG) dafür ein, dass die Tarife den hohen schweizerischen Qualitätsansprüchen angepasst bleiben oder werden. Das Labor ist ein unverzichtbares Instrument für die gute Medizinpraxis. Wirtschaftlich gesehen führen rasch verfügbare Resultate zu signifikanten Kosteneinsparungen.

Unterstützen Sie daher die Bemühungen der FMH und der Kollegen, die sich auf diesem Gebiet für den Erhalt eines Praxislabors einsetzen, das sich durch hohe Qualität auszeichnet und für alle Beteiligten rentabel ist. 